\title{
Factors Contributing to Community None Acceptance for Indoor Residual Spraying (IRS) for Malaria prevention in Chipushi Area of Mpika, Zambia
}

\author{
Tembo Elemiya \\ Master of Public Health, Texila American University, Zambia
}

\begin{abstract}
Indoor residual spraying (IRS) is one of the primary vector control interventions for reducing and interrupting malaria transmission. In recent years, however, it has received relatively little attention. Just like it is the case in the 17 villages of the Chipushi area of Mpika district in Muchinga province, Zambia. In community work, among the things that attracted my attention are the non-acceptance and perceived negative attitudes towards acceptance for indoor residual spraying. The purpose of this study was to analyze and establish the factors that contribute to none acceptance for Indoor Residual Spraying (IRS) in the Chipushi area of Mpika, Zambia. None acceptance, in this case, refers to a widespread negative response to having indoor residual spraying performed in people's homes at the community level. Simple random sampling was used to come up with the study population. And after executing the study through the questionnaire and semi-structured interviews on the 97 respondents, findings revealed that $12(12.4 \%)$ respondents believed that chemicals used in IRS increase mosquitoes and bed bugs instead of controlling them. Other $22(22.7 \%)$ never believed IRS could reduce the incidence of malaria. $18(18.6 \%)$ respondents avoided being in-convenience by the spray operators and health personnel. $10(10.3 \%)$ respondents don't want to be seen how wrecked their houses are or fear of being embarrassed due to lack of sanity in their homes. Similarly, 17 (17.5\%) respondents believed that by the culture, they could not allow strangers to expose their privacy in their homes. Surprisingly $26(26.8 \%)$ respondents never just wanted to participate in spray operations.
\end{abstract}

Keywords: Community, Households, Indoor Residual Spraying, Malaria elimination, Prevention.

\section{Introduction}

Malaria is one of the leading causes of mortality, particularly in Africa, with approximately $90 \%$ of the 528,000 malaria deaths estimated all over the world occurring in Africa. Another region of interest, while recognized internationally for its ambitious goal and plans of malaria elimination and having attained pre-elimination levels, the Southern Province of Zambia as a whole has surprisingly remained a highly endemic area for malaria. This is my major concern being a citizen of the country Zambia. For the past one year, we have seen that malaria still tops on the top ten diseases in the Mpika district of Muchinga Province, Zambia [1].
The national malaria profile in Zambia shows an increased risk for malaria, with almost 16 million people at risk. In 2015 alone, it was estimated that over 5 million malaria cases (about 98\% is due to plasmodium) were seen and recorded [1].

My objectives for this study are actually aimed at laying down the foundation for replicating new research.

\section{General Objectives}

1. What are the factors contributing to none acceptance for IRS in communities?

2. What strategies could be employed to promote acceptance for IRS? 


\section{Specific Objectives}

1. To ascertain the cause for none acceptance for indoor residual spraying in the Chipushi area of Mpika, Zambia.

2. What is the reason for none acceptance for IRS in the Chipushi area of Mpika?

The table below shows the malaria incidence in Zambia as for the 2015 fiscal year. The outpatient cases reported show that the high cases were reported in the copper belt province while lower cases were reported in the southern province. While high incidences per 1000 cases were recorded in Northwestern, Luapula, and Muchinga Provinces. My study area of Chipushi is actually in the Muchinga Province.

Table 1. Malaria incidence in Zambia

\begin{tabular}{|l|l|l|l|}
\hline Province & Population 2015 & $\begin{array}{l}\text { Total malaria OPD } \\
\text { cases reported }\end{array}$ & $\begin{array}{l}\text { Total reported malaria } \\
\text { incidence per 1000 }\end{array}$ \\
\hline Copper belt & $2,362,216$ & 815,988 & 345.4 \\
\hline Luapula & $1,102,625$ & 773,710 & 701.7 \\
\hline Northwestern & 843,156 & 680,375 & 806.9 \\
\hline Eastern & $1,813,450$ & 670,838 & 369.9 \\
\hline Northern & $1,304,434$ & 608,925 & 466.8 \\
\hline Western & 993,472 & 496,326 & 499.6 \\
\hline Muchinga & 449,445 & 449,445 & 502.1 \\
\hline Lusaka & $2,943,695$ & 92,185 & 31.3 \\
\hline Southern & $1,714,787$ & 45,794 & 26.7 \\
\hline
\end{tabular}

Source: HMIS 2015 OPD daily

Indoor Residual Spraying (IRS) is simply one of the very primary vector control measures or interventions intended for reducing and interrupting malaria transmission via a mosquito bite. Unfortunately, in recent years, however, it has received relatively little or sub-standard attention [2].

By definition Indoor Residual Spraying (IRS) is simply the application of somewhat a longacting chemical insecticide on the walls and roofs of all houses and domestic animal shelters in a given area in order to kill the adult mosquitoes that lands and rests on these surfaces of the walls [2].

The government of the Republic of Zambia (GRZ) resumed the IRS programs in 2003, and then in recent years, it has been increasing resource allocation towards malaria control in general, and IRS, in particular, is high on budget. PMI had supported IRS since 2009 and was preceded by three years of USAID- funded IRS. By 2015, PMI was supporting IRS operations in 25 PMI focus districts in Zambia. Since 2016,
Zambia has benefitted from the UNITAIDfunded NgenIRS project and subsidy also, and in 2017, the PMI- supported campaign targeted 648,800 structures in the same four high burden provinces and aimed to protect a population of $2,626,718$, which translates to almost $15 \%$ of the population. PMI plans to spray around 500,000 structures in the 2020 season alone, of which data is yet to be verified. District Health Offices (DHOs) provide overall planning, conclusion, and monitoring of malaria activities within their districts. Activities such as the implementation of IRS, ITN distribution, and malaria case management at level one hospitals, health centers, and in the community are implemented through DHOs. The National Malaria Elimination Center (NMEC), previously known as the National Malaria Control Center (NMCC), provides technical support but not operational assistance at these levels [3].

Under the district health office in my study area of Mpika in Zambia, malaria is still ranked the highest cause of morbidity and mortality. 
And Muchinga province, under which Mpika falls, is ranked among the top four most affected provinces in Zambia. Malaria disease caused by Plasmodium falciparum may result in death within hours or a few days of infection, especially in those with low immunity, such as children, pregnant women, people living with HIV/AIDS, and travelers [4].

Studies have shown and explored some factors hindering the acceptability and use of available malaria prevention measures to better inform area-specific strategies that can lead to the much-needed malaria elimination levels [5].

\section{Methods}

Understanding approach to research has a number of advantages which makes it suitable and more reliable to be used. Basically, it provides strengths that rule out or offset the weaknesses of both quantitative and qualitative research [6]. For instance, it is clear that quantitative research is very weak in understanding the context and setup in which people tend to behave, something that qualitative research resolves. On the other hand, qualitative research is seen as deficient because of the potential for biased interpretations made by the researcher and the difficulty in generalizing findings to a large group. Quantitative research does not have these weaknesses. Thus, by using the understanding approach to research, the strengths of each approach can make up for the weaknesses of the other.

Additionally, it was pointed out further that the understanding approach provides a more complete and comprehensive understanding of the research problem than either quantitative or qualitative approaches alone. Moreover, it provides an approach for developing better, more context-specific instruments. By the way, using qualitative research makes it possible to gather information about certain topics or the construct in order to develop an instrument with greater construct validity, that is, the measures the construct intends to measure after all. And above all, this, in turn, helps to explain findings or how the causal processes work [6].

However, it is stipulated that an understanding approach to research has as well a number of disadvantages or demerits [7]. For instance, the research design can sometimes be very complex. In addition to that, it takes much more time and resources to plan and implement this type of research and therefore requires added inputs from stakeholders. Moreover, it may be difficult to plan and implement one method by drawing on the findings of another. And also, it may be unclear how to resolve clear discrepancies that arise in the interpretation of the findings, and hence solutions are found.

Constraints are common in research especially doing in rural areas like in my place. By definition, constraints are obstacles or even barriers that prevent or hinders progress. Thus, when planning well-meaning research, it is important to take into consideration such constraints in research that could hinder progress. The first practical constraint is the lack of cooperation or insecurity attitudes by some respondents that the researcher targets. This may make it hard to collect enough and quality data.

In addition, accessing locally published literature may also be another challenge, especially in under privileged communities like my own study area. Moreover, time is another constraint because it could be insufficient to conduct the needed experiments, so that the duration may take much longer than the usual period of any research grant or resources (for instance, the project needs more than 5 years of support to be completed) [8].

According to verified reports, the other practical constraint is that the required research experiments may be deemed to be too dangerous to be conducted at a given institution or facility [9]. Lastly, the proposed experiment will necessitate the violation of some official prohibitions, restrictions, or public laws which are established by the national government agencies, for example, DNA cloning, stem-cell 
studies, chemical synthesis of some toxic materials, among others.

Therefore, planning the research approach should take this practical constraint into consideration in order to determine which approach could be suitable.

The research process takes a number of steps for it to be developed in its complete manner. The first step is to identify and develop your topic. The second step is to find background information. The practice is that one needs to look up for keywords in the indexes to the subject encyclopedia. Reading articles in this encyclopedia will set the context for the research. You need to take note of any relevant items in the bibliography right at the end of the encyclopedia articles. Additional background information may be found in your lecture notes, textbooks, and reserve readings [10].

Thirdly, use catalogs to find books and media. In the fourth step, you use indexes to find periodical articles that is use periodical indexes and abstracts to find citations to articles. The indexes and abstracts may mostly be in hardcopy print or softcopy computer-based formats or even both. One can easily find periodical articles by the article authors, title, or keywords by using the periodical indexes available in the library's home page part [11].

The fifth step is to find additional internet resources. Almost everyone now is aware of the uses of the Google platform and its accessories, Google Scholar, Google Books, Google News, YouTube, among others, to search and find information on the open Internet so fast (as opposed to the subscription-only resources you will encounter in steps 2 through 4 above).

The Sixth step involves evaluating what you find, See, and how to critically analyze the information sources to distinguish scholarly from Non-Scholarly Periodicals. If you have found too many or too few source materials, you may have to narrow or broaden your topic to suit the search.

The last step, as stated in other reports, it is cited that what you find using a standard format as prescribed by the faculty in which the study is directed. It is worth noting that knowingly presenting the work of other people or scholars as your own work is known as plagiarism [11].

\section{Study Area}

The study will focus on the seventeen villages in the Chipushi area of Mpika, Zambia, with a total catchment population of approximately 4494. These villages are based in the Chishibesonde ward of Mpika Central constituency. Villages covered include;

Kabuswe, Kabimbili, Mashinda, Kamfwa, Lupasha, File, ZNS, Matebe, Mama, Kansumba, chailo, Mungalaba, Mubanga, Mwiko, Kanungulila, Shipili and Namfunda. Much of the studies will involve focused group discussions and questionnaires to be administered to selected individuals in the sample group, with the majority of the inhabitants of chipushi living in extreme poverty.

\section{Study Population}

The study population will include the target population from the Chipushi area. The population of interest are derived from the sampled households out of the 926 households doted around the catchment area. All these are selected from all the 17 target villages in the Chipushi catchment area.

\section{Study Duration}

The study is expected to take approximately six to nine months. However, with the help of community involvement, it is possible to have the final report before the estimated time frame. I have made a very good personal relationship with the community structures in all the study areas of Chipushi hence the advantage of not pressurizing while collecting essential data. This co-existence with the community is very important in order to win the support of the sample population as well as the community leadership, who in turn give massive support to data collection needs. This collaboration helps to shorten and simplify the process of research. 


\section{Sampling Method}

In this study, a simple random sampling technique was used to select community respondents while judgmental, also called as purposive sampling, was deployed to select some respondents like the healthcare workers and 17 headmen. Simple random sampling was picked because of its advantage. Sample random sampling gives each participant in the population an equal chance to be part of the sample of the study. In other words, simple random sampling does not bias. On the other hand, judgmental sampling has been chosen because it produces a sample that is more representative of the population [12].

\section{Sample Size}

The study sample size will include 20 neighborhood health committee members, 20 household heads, 20 health care workers, 20 youths, and 17 headmen. The total sample of 97 respondents. All these are selected from all the 17 target villages in the Chipushi catchment area. The youths are involved because it's a very aggressive group in the community, and they have information concerning the happenings in the community. Omitting them may mean missing out on the vital voice's cardinal to the study.

Study tools various schedules, checklists, etc. to be used.

In obtaining information for the study, the following tools (instruments) were used: questionnaire, observation schedule, and semistructured interviews guide.

\section{Questionnaires}

The questionnaires were used because of the following advantages that they gave to the researcher: it was easy to collect data from a large sample, and because of this result can be dependable and reliable, confidentiality was upheld and saved time. Furthermore, the questionnaire made it possible to reach respondents in a more convenient way, and above all, it was free from the bias of the interviewer. However, it also had some disadvantages, and these include: it was likely to be the slowest of all because it allowed respondents to answer questions on the questionnaire at their own pace. It sometimes results in the low rate of return of the duly filled questionnaire.

\section{Focused Group Discussion Schedule}

The researchers chose this tool because it gave him first-hand information that he used to complement other methods. The respondents are put together in small groups and share the topic for discussion. A guided approach is the best in this type of data collection especially dealing with less literate people.

\section{Semi-Structured Interview}

A semi-structured interview was selected to be used in collecting data due to the following advantages; firstly, data collected in this manner are suitable for interpretative phenomenological analysis (IPA); the process is also very fast. However, it is not free from biasness. This was taken care of by avoiding bias.

\section{Results}

My critical discovery about this research is that most people in the study population are generally illiterate and poor. They are mostly guided by their social norms and traditions, which are perceived to protect their integrity. These traditions come from way back in ancient times or are sometimes wrongly oriented. Going down to dig the roots of these misconceptions may be the very solution to reducing the cases of malaria in the long run. The demographic characteristics of the respondents of this study are mostly community members who live together under similar conditions. 


\section{Questionnaire Respondents by gender}

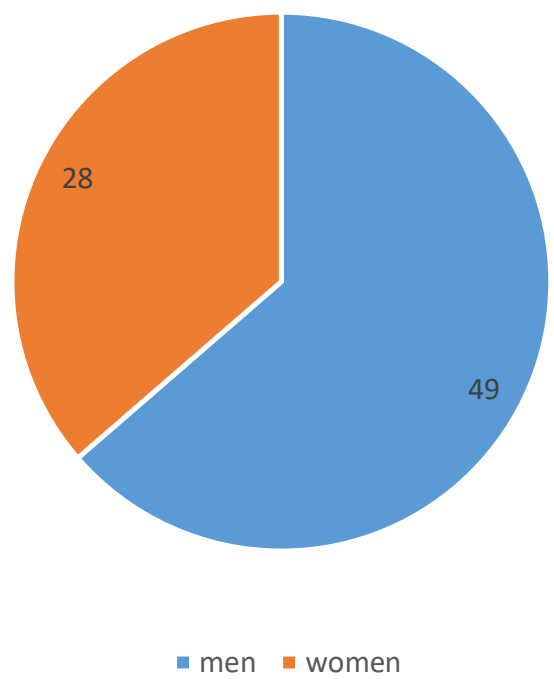

Figure 1. Demographic Characteristics of Respondents by Gender

It is one thing to say that research should be balanced on sexual grounds due to issues of gender balancing. However, the best practices on the selection of the sample size will mostly come out in a random way. After selection of my simple random selected sample, I found that men out-numbered women by double. However, it is manageable within the context of the study. This is because most homes are male-headed, unlike otherwise.

\section{Respondents by Status}

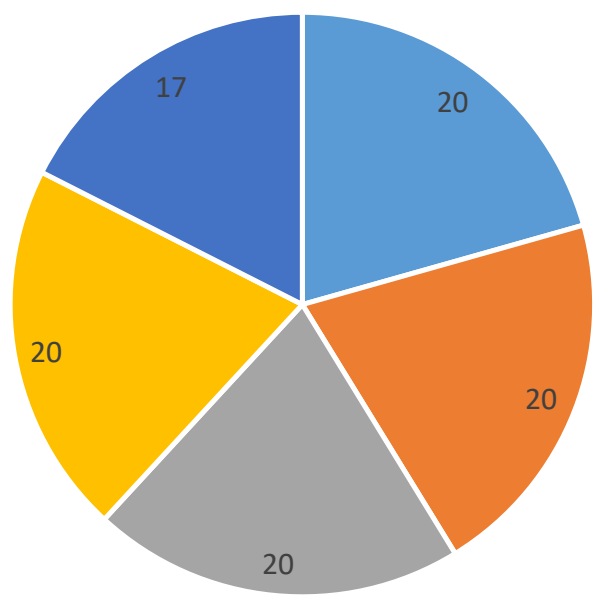

- Healthcare workers

- Household heads

$\because$ NHCs

- Youths

- village heads

Figure 2. Demographic Characteristics of Respondents by Status 


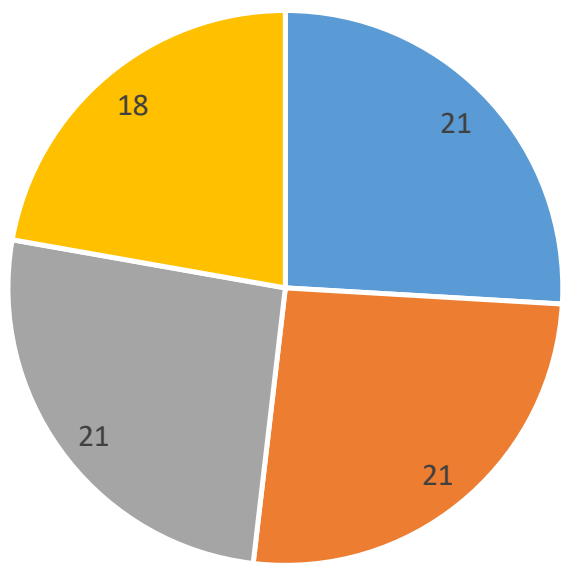

- HCWs $\square \mathrm{HHHS}=\mathrm{NHCs} \backsim$ Village Heads

Figure 3. Demographic Characteristics of Respondents by Percentage

The other findings concerning Indoor Residual Spraying in some areas of Mpika rural shows that the past three IRS operations did not achieve its intended targets. The entire district recorded almost $45 \%$ plus coverage. It is much less in my study population area of Chipushi. Being a primary health care provider working in a small clinic in Chipushi, Mpika, I have personally seen and experienced the apathy exhibited by the communities in accepting IRS. IRS is inherently challenging, and each year has brought its share of problems [13].

From my own observation, I saw how some people underplayed the process of IRS and ending up running away from the spray operators to the woods. Others leave children to report to the spray operators that they are not home when they actually are inside the house, hiding. Some villages preferred to go in their farms to work than waiting for their homes to be sprayed. Even after advertising and sensitization on the value of spraying, people never understood the importance thereof. The value of which is less received in the communities under this study. Probably future research should target to evaluate the effectiveness of IRS on malaria vectors, prevalence, and the community perception on IRS after spraying. Other studies believe that larval source management has several aspects that are significantly more sustainable than IRS and ITNs [14]. However, World Health Organization recommends the use of ITNs and IRS as part of a global framework for integrated vector management as the mainstay of vector control in sub- Saharan Africa [15].

\section{Discussion}

My research question, 'Factors Contributing to Community none acceptance for Indoor Residual Spraying (IRS) against mosquitoes as prevention for malaria in rural areas of Mpika, Zambia. I endeavor to find out why there is little acceptance in IRS in rural areas of Mpika, Zambia. From the primary survey I did in almost 17 villages, there are a number of concerns regarding IRS.

In the recent past, there has been poor coverage of indoor residual spraying in most rural parts of Mpika, Zambia. There is a need to do research in this area to ascertain why the acceptance levels for IRS are low in these rural communities. 
This study will help authorities and communities to adjust and understand the benefits of indoor residual spraying in the prevention of malaria. Without this, the battle for the elimination of malaria by 2021 will be farfetched. The control for malaria is a complex chain of measures that often complement one another [16].

As a public health professional, I am alive to the fact that the fight for the elimination of malaria in our communities, especially in subSaharan Africa and Zambia in particular, is supposed to receive the attention it deserves. I really want to understand the development of the effect of IRS in the prevention of escalating cases of plasmodium in the communities as a positive stride towards winning the fight against malaria.

The vital information worth knowing is that there are well above 16 million people who are at high risk of malaria infection in Zambia. And it was estimated in 2015 that there were over 5 million malaria cases. This is extremely high.

If knowledge of the effects and indeed the benefits of accepting IRS is optimized in rural communities, we would one day very soon defeat malaria. This work is targeting pulling out the misconceptions, negative social norms, beliefs, myths, and any other negative orientation surrounding the Indoor Residual Spraying programs.

In my work, there will be no clinical or chemical experiments involved to ascertain the efficacy and effect of the chemicals used during indoor residual spraying, as this will require high-level laboratory experiments and tests. However, necessary information concerning chemicals used will be collected in order to understand what other literature reports concerning these chemicals.

This will help us find out how these chemicals can affect individuals as well as the environment. Maybe the study could also make us learn how these chemicals could be a threat to health in another way. Or could this be the reason why communities have a negative response towards Indoor Residual Spraying? This is the question that begs to be answered if progress in the malaria fight is to be made in the area of prevention. As it is said, prevention is better than cure. In our local dialect, it is said that "the earliest person drinks cleaner water." Meaning whoever starts the race early will definitely find a winning foot. The earlier you act, the better. However, the target and sample population involved in the study is unfortunately comprised of mostly less learned people, although they have a wide range of perceived knowledge in their local beliefs and customs. Almost 90\% of the target population are peasant farmers depending mainly on seasonal crops like maize, pumpkins, and sweet potatoes, mostly planted in the rain season.

The recent call for malaria elimination is expected to result in changes in the selection of interventions, and additional research into uses of environmental management in varied settings is listed as necessary for improving vector control [17]. Our primary goal for this study is to get verified information why there is poor acceptance for indoor residual spraying in some rural areas of Mpika, Zambia. This research will be helpful to many more communities in our country, Zambia and beyond, to come up with measures that will promote acceptance. Information will also be shared with the concerned communities in the study and any other interest groups. Many communities involved are in under privileged settings of rural Mpika.

However, the main concern is that they are also vulnerable to various diseases, which include malaria. Finding lasting solutions to reducing malaria can greatly help in reducing morbidity and mortality from malaria. Moreover, malaria is the highest-ranked cause of morbidity and mortality in this area of study.

All avenues to eliminate malaria require the involvement of the communities where cases of malaria come from. Without considering inputs from the community can be a grave omission in the fight for a malaria free society. Hence the 
significance of such a study to be supported and be actualized. Indoor Residual Spraying, if well implemented, will be a major force in the elimination of malaria. I am passionate about this study because malaria is still our number one cause of morbidity and mortality in all ages in our country, Zambia.

Indoor Residual Spraying (IRS) with insecticides is a very powerful way to rapidly reduce malaria transmission by spraying inside homes. It involves mostly the spraying of the inside walls of housing structures with a certified chemical or insecticide. Typically, once or twice per year. To confer significant community protection, Indoor Residual Spraying (IRS) should be implemented at a high level of coverage in areas where the activity is being done. Globally, IRS effective protection declined from a high of 5\% in 2010 to about $3 \%$ in 2017, with decreases seen across all World Health Organization regions [18].

\section{Conclusion}

A number of malaria prevention strategies have been evaluated since the turn of the century. [19]. However, there are failed programs in malaria prevention in many communities of Mpika, Zambia, for a long time. The average coverage for the most recent two Indoor residual sprayings (IRS) in the Chipushi area of Mpika were very alarmingly low. Records for the past three years at the nearby catchment area health facility shows that in the first operation in 2017, the coverage for IRS was 122 (13.2\%), 199 (21.4\%) in 2018 out of the 926 households where sprayed. In the most recent campaign in 2019 , only 88 (9.5\%) households were sprayed out of the 926 households.

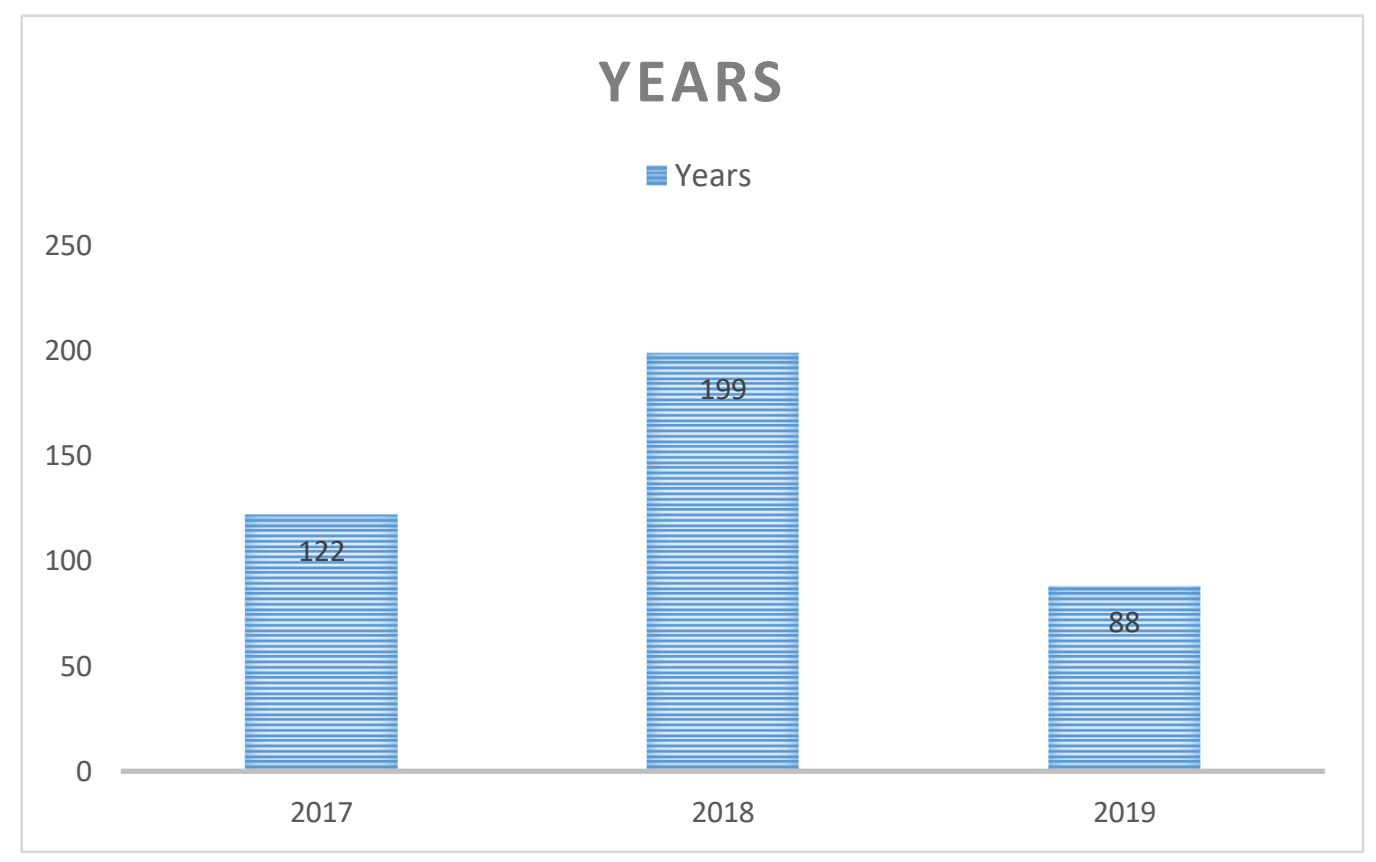

Source: HMIS, 2020 [1]

Figure 4. Health Facility Three Years' Trend Data for IRS- Chipushi Area

This trend in the Chipushi area of Mpika, Zambia, means that the acceptance levels for IRS fluctuate. This is not good for coverage and meeting the targets for the program of malaria prevention. If this trend is left unchecked, then the fight for a malaria-free society will be farfetched. This trend from 2017 to 2019 is worth being investigated and possible reasons established for the poor coverages recorded in achieving the full targets. The findings, recommendations, and conclusions thereof will most likely help to improve the intended targets.

The other findings concerning Indoor Residual Spraying in some areas of Mpika rural 
shows that the past three IRS operations did not achieve its intended targets. The entire district recorded almost $45 \%$ plus coverage. It is much less in my study population area of Chipushi. Being a primary health care provider working in a small clinic in Chipushi, Mpika, I have personally seen and experienced the apathy exhibited by the communities in accepting IRS. As a matter of fact, IRS is unfortunately challenging, and each year brings with it various problems.

From my own observation, I saw how some people demonized the process of IRS and ending up running away from the spray operators to the woods. Others leave children to report to the spray operators that they are not home when actually are inside the house, hiding. Some villages preferred to go in their farms to work than waiting for their homes to be sprayed. Even after advertising and sensitization on the value of spraying, people never understood the importance thereof. The value of which is less received in the communities under this study. Future research should probably target evaluating the effectiveness of IRS on malaria vectors, prevalence, and the community perception on IRS after spraying. On the other hand, other people never just accepted to have their houses sprayed.
After executing the study through the questionnaire and semi-structured interviews, findings of the research revealed that 12 of the respondents believed that the chemicals used in IRS increase mosquitoes and bugs instead of controlling them. Yet other 22 never believed IRS could reduce the incidence of malaria in the communities.

In this study, I also found that 18 respondents avoided being in-convenience by the spray operators and other health personnel. In the same vain, 10 respondents don't want to be seen how wrecked their houses are. For fear of being embarrassed due to lack of sanity in their homes. Yet again, it was found that 17 of the respondents believed that by the culture they could not allow strangers to expose their privacy in their homes. Others believed that it was embarrassing to see that the spray operators were too young to see their privacy.

One surprising finding was that 26 respondents never just wanted to participate in the spray operations. These did not give any reasons for not allowing spray operators to cover their homes. They participated in the enumeration process but never took part in the final spraying process.

The following table/figure shows how the distribution of respondents was recorded.

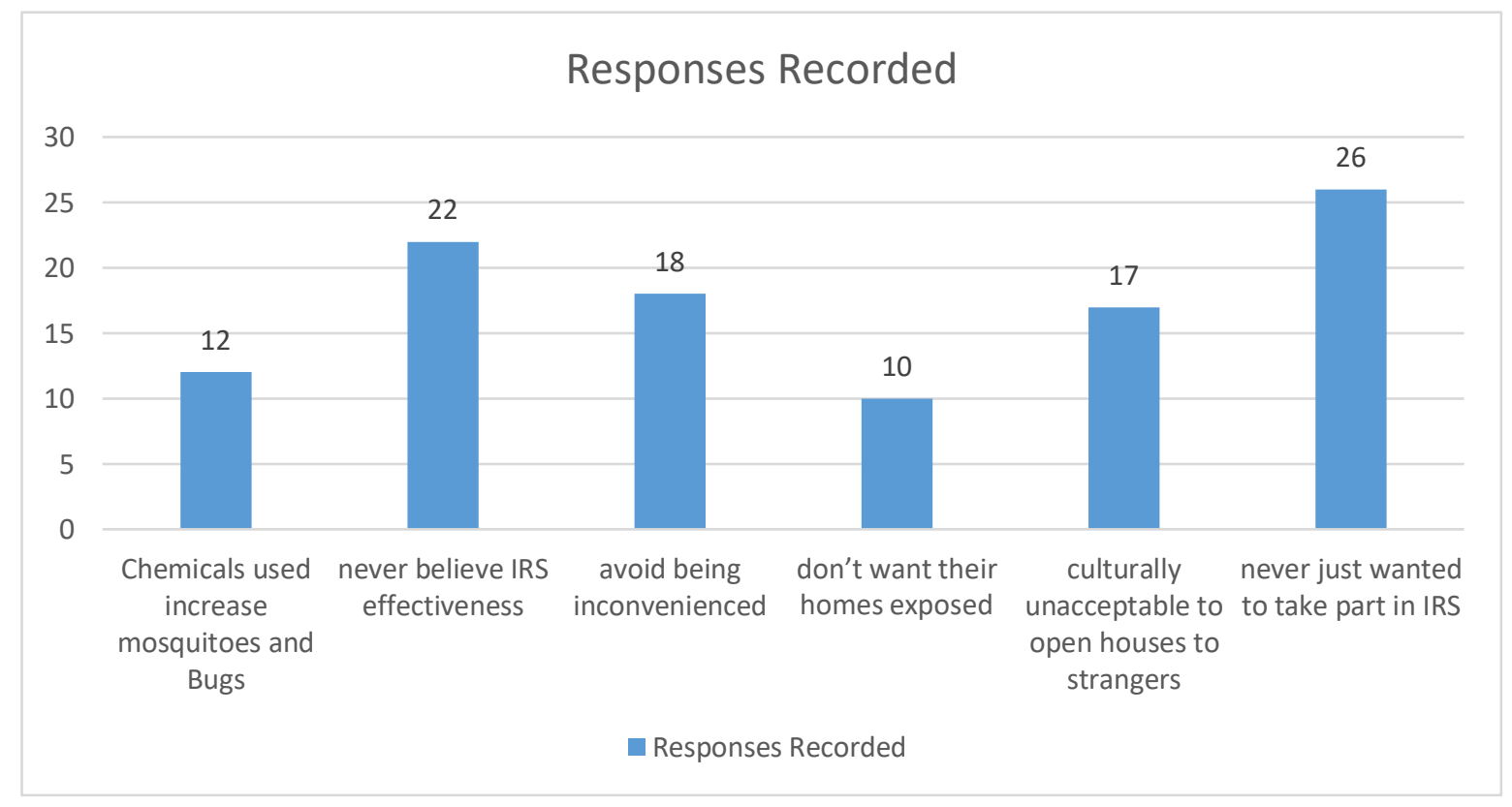

Figure 5. Respondents' Distribution of Responses 
This study will mainly focus on the factors behind none acceptance of the Indoor Residual Spraying in the rural chipushi area of Mpika, Zambia. And this will give insight into further research in the responses recorded thus far.

When enacting a large-scale new measure such as IRS to curb incidence of disease, Gamiccia suggests drawing up a health education plan that recognizes community priorities and has inputs from an epidemiologist, sociologist, and health educator working together [20]. Malaria eradication is a goal worth pursuing today more than ever before [21].

\section{Conflict of Interest}

After a thorough interaction with my guide concerning conflict of interest in this study, I was able to identify and mitigate potential or actual conflict of interest and are managed accordingly. Being a qualified health worker was easy to interview my fellow health workers using a questionnaire to eliminate cohesion and possible bias. Very interesting and challenging all together.

\section{References}

[1] Health Management Information System., 2020, Health statistics: Information office, 8(2), 36-42, https://www.zambiahmis.org/dhis.

[2] World Health Organization., 2006, Indoor Residual Spraying: Use of indoor residual spraying for scaling up global malaria control and elimination, 12(6), 1- 8,

https://apps.who.int/iris/handle/10665/69386.

[3] US President's malaria initiative., 2019, Zambia malaria operational plan FY 2020: USAIDS, Global malaria strategy, 12(4), 39-46, https://www.pmi.gov. [4] Zambia Malaria Elimination Centre., 2015, Malaria Overview: Malaria Elimination Strategy, https://www.nmec.org.zm/malaria-overview.

[5] Ingabire, C.M. et al., 2015, Factors impeding the acceptability and use of malaria preventive measures: implications for malaria elimination in eastern Rwanda, $14: 136$, $1-7$, https://www.researchgate.net/publication/274707254

\section{Acknowledgements}

First and foremost, I would like to thank my heavenly father, Jehovah, for his unfailing love and protection for my life. I would not be here if not for him. It has been a tough but an interesting journey at Texila. Many well-meaning academicians, faculties, and coordinators helped me to achieve my goal today. I am grateful for their immeasurable commitment and encouragement.

Furthermore, I thank my student mentors from year one to date for their resilient support. I cannot forget my employers for allowing me time offs to attend teachings, webinar sessions, conferences, and exams with no objection. I am highly indebted to them for their understanding. I cannot forget to acknowledge my district health office for their technical support and my guide, Prof. Gelson Tembo. Finally, I thank my family, who have been helpful throughout my studies. To all of these wonderful people am thankful and excited for being my family.

_Factors_impending_the_acceptability_and_use_of_ malaria_preventive_measure_implications_for_mala ria_elimination_in_eastern_Rwanda.

[6] Davis, B. G., 1993, The Understanding Approach to Research. San Francisco: Jossey-Bass.

[7] Altman, H. B., \& Cashin, W. E., 2003, The Research Methodology. London: McMillan Press.

[8] Eberly, M.B., 2001, Research practical obstacles: Journal of research, 50(1), 6- 7.

[9] Daniels, C.K., 1988, The research constraints: Bolton, MA, Anker.

[10] Woolcock, M.J., 2003, The research steps: Journal of research project, 12(2), 1- 9, https://www.iedunote.com/research-process.

[11] Grunert, J., 1997, The seven key Research steps: Bolton, MA, Anker, https://course.lumenlearning.com/ivytecheng111/chapter/the-seven-steps-of-the-researchprocess-revise-customize/. 
[12] N'gandu, S.K., 2013, writing a research proposal in educational research, Lusaka: The University of Zambia.

[13] US presidential malaria initiative., 2019, Zambia malaria operational plan FY 2020: US AID- Global malaria strategy, 51- 52, https://www.pmi.gov/where-we-work/zambia.

[14]Lindsay, S.W. \& Fillinger, U., 2011, Larval Source management for malaria control in Africa: myths \& reality. Malaria journal, 10:383, 5-6, https://www.malariajournal.com/content/10/1/353.

[15] Majambere, S. et al., 2010, Is mosquito larval source management appropriate for reducing malaria in areas of extensive flooding in the Gambia? A crossover intervention trial. American Journal for tropical medicine \& Hygiene, 82(2), 176-184.

[16] Steketee, R.W., 2009, Control of malaria: American journal of tropical medicine \& hygiene. 80(6), 879-880,

https://www.ajtmh.org/cgi/reprint/80/6/879.

[17] Castrol, M.C., et al., 2009, community based environmental management for malaria control: evidence from a small- scale interaction in Dar es Salaam, Tanzania. Bio-med central malaria journal, $8: 57$,

https://www.malariajournal.com/content/8/1/157.

[18] World Health Organization., 2015, malaria: WHO global technical strategy for malaria 20162030, https://reliefweb.int/report/world/who-globaltechnical-strategy-malaria-2016-2030.

[19] Seidlein, L.V., 2016, Malaria prevention strategies: $17^{\text {th }}$ international congress on infectious diseases. International journal of infectious diseases, $45 \mathrm{~S}$ $1-477$, http://dx.doi.org/10.1016/j.ijid.2016.02.069.

[20] Ganiccia, G., 1981, Health education in malaria control: Why has it failed, world health forum, 2, 385393.

[21]Hall, B.F. \& Fauci, A.S., 2009, Malaria control, elimination, \& eradication: The role of the evolving bio-medical research agenda. The journal of infectious diseases, 200:1639-43, https://academic.oup.com/jid/articleabstract/200/11/1639/831923. 